\section{Morphological and ultrastruc- tural analysis of normal, injured and osteoarthritic human knee menisci}

\begin{abstract}
Michela Battistelli, ${ }^{1}$ Marta Favero, ${ }^{2}$ Debora Burini, ${ }^{1}$ Giovanni Trisolino, ${ }^{3}$ Dante Dallari, ${ }^{4}$ Lucia De Franceschi, ${ }^{4}$ Steven R. Goldring, ${ }^{5}$

Mary B. Goldring, ${ }^{5}$ Elisa Belluzzi, ${ }^{2,6}$ Giuseppe Filardo, ${ }^{7}$ Brunella Grigolo, ${ }^{8}$ Elisabetta Falcieri, ${ }^{1}$ Eleonora Olivotto ${ }^{8}$

${ }^{1}$ Department of Biomolecular Sciences, Urbino University "Carlo Bo", Italy ${ }^{2}$ Rheumatology Unit, Department of Medicine (DIMED), University Hospital of Padua, Italy

${ }^{3}$ Pediatric Orthopedic and

Traumatology, IRCCS Istituto

Ortopedico Rizzoli, Bologna, Italy

${ }^{4}$ Reconstructive Orthopedic Surgery

Innovative Techniques - Musculoskeletal

Tissue Bank; Revision surgery of hip patients with late-stage OA, showing disorganization of collagen fibers and increased proteoglycan content. Cells of healthy menisci showed mainly diffuse chromatin and well preserved organelles. Both in traumatic and in OA menisci, we observed increased chromatin condensation, organelle degeneration, and cytoplasmic vacuolization, a portion of which contained markers of autophagic vacuoles. Areas of calcification were also observed in both traumatic and OA menisci, as well as apoptotic-like features that were particularly prominent in traumatic meniscal tear samples. We conclude that meniscal tissue from patients with traumatic meniscal injury demonstrate pathological alterations characteristic of tissue from older patients undergoing TKR, suggesting that they have high susceptibility to develop OA.
\end{abstract}

prosthesis and development of new implants, IRCCS Istituto Ortopedico

Rizzoli, Bologna, Italy

${ }^{5}$ Hospital for Special Surgery, New York,

NY, USA

${ }^{6}$ Musculoskeletal pathology and

Oncology Laboratory, DISCOG,

University of Padua, Italy

${ }^{7}$ ATRC-Laboratory, IRCCS Istituto

Ortopedico Rizzoli, Bologna, Italy

${ }^{8}$ RAMSES Laboratory, RIT

Department, IRCCS Istituto Ortopedico

Rizzoli, Bologna, Italy

\begin{abstract}
The human meniscus plays a crucial role for transmission and distribution of load across the knee, as well as shock absorption, joint stability, lubrication, and congruity. The aim of this study was to compare the complex geometry, unique ultrastructure and tissue composition of the meniscus in healthy (control) and pathological conditions to provide understanding of structural changes that could be helpful in the future design of targetted therapies and improvement of treatment indications. We analyzed meniscus samples collected from 3 healthy multi-organ donors (median age, 66 years), 5 patients with traumatic meniscal tear (median age, 41 years) and 3 patients undergoing total knee replacement (TKR) for end-stage osteoarthritis (OA) (median age, 72 years). We evaluated the extracellular matrix (ECM) organization,
\end{abstract}

the appearance and distribution of areas of calcification, and modifications of cellular organization and structure by electron microscopy and histology. The ECM structure was similar in specimens from traumatic meniscus tears compared to those from

\section{Introduction}

In the recent decades extensive studies have demonstrated the importance of meniscus integrity in preserving knee function and stability, ${ }^{1}$ as well as the association of meniscal damage with development and progression of osteoarthritis (OA). ${ }^{2}$ Both traumatic and degenerative meniscal changes are associated with elevated risk of $\mathrm{OA},{ }^{3,4}$ but the risk is greater after degenerative-type tears. ${ }^{5}$ Meniscal degeneration, which can also occur in both young and older people, is defined as structural and functional failure of the tissue and it is caused by various factors such as repetitive trauma and joint malalignment. ${ }^{6}$ Aside from age, gender, body mass index (BMI), and sports activities, multiple studies have indicated that a longer interval between anterior cruciate ligament injury and surgical reconstruction increases the risk of meniscal degeneration and tears.

Pauli et al. ${ }^{8}$ found several differences that distinguish meniscus degeneration caused by aging versus progressive OA. The major changes in aged menisci are increased Safranin O staining intensity, decreased cell density, and mucoid degeneration associated with loss of collagen fiber organization. ${ }^{8}$ These age-related meniscal tissue degeneration changes, even in the absence of overt tears is associated with molecular and cellular alterations indicative of processes characteristic of cellular senescence. ${ }^{9}$ Additional alterations are observed in advanced OA, including severe fibrocar-
Correspondence: Michela Battistelli, University of Urbino "Carlo Bo", Via Ca' le Suore 2, 61029 Urbino (PU), Italy.

Tel. +39.0722.304269

E-mail: michela.battistelli@uniurb.it

Key words: Meniscus; meniscal tear; osteoarthritis; transmission electron microscopy; extracellular matrix degeneration.

Contributions: EO, MF, MB, study concept and design; GT, arthroscopic and THR surgeries; EO, histology and immunohistochemistry experiments; MB, TEM analysis; EO, MB, data acquisition; LDF, multiorgan donor samples collection; EO, MF, MB, GT, substantial contribution to data analysis and interpretation; GF, contribution to patient selection, sample collection; EO, MF, GT, MB, GF, EF, $\mathrm{BG}, \mathrm{MG}, \mathrm{SG}$, contribution to manuscript editing. All the authors participated in drafting the article or in providing critical revisions for important intellectual content and gave the final approval of the version to be published.

Conflict of interest: The authors report no conflicts of interest related to any aspects of the presented manuscript. The authors alone are responsible for the content and writing of the article.

Funding: The study was supported by Ministero della Salute - GR-2010-2317593, CARISBO Foundation (Bologna, Italy) and University of Urbino "Carlo Bo", Italy.

Received for publication: 13 November 2018 Accepted for publication: 11 January 2019.

This work is licensed under a Creative Commons Attribution-NonCommercial 4.0 International License (CC BY-NC 4.0).

(C) Copyright M. Battistelli et al., 2019

Licensee PAGEPress, Italy

European Journal of Histochemistry 2019; 63:2998 doi:10.4081/ejh.2019.2998

tilaginous disruption, fine fibrillations, and loss of structure. Of interest, biomechanical and functional properties and, consequently, the resistance to injuries are dependent on the meniscal integrity with no reported difference observed between degenerative and traumatic meniscal tear. ${ }^{10}$

The meniscus sustains many different forces such as shear, tension, and compression. It also plays a crucial role in load bearing and transmission, shock absorption, as well as lubrication and nutrition of articular cartilage. These multiple functions are supported by the extracellular matrix (ECM) components and the activities of the resident cell populations. ${ }^{1}$

The aim of this study was to investigate ECM composition and cell morphology of normal, injured, and $\mathrm{OA}^{11-13}$ menisci from 
human knees employing transmission electron microscopy (TEM). Possible correlations between meniscal integrity and duration of symptoms after meniscal injury were investigated to understand structural changes, which could be helpful to design future targetted therapies and improve treatment indications.

\section{Materials and Methods \\ Patient recruitment, clinical data, and sample collection}

This work was designed as an observational study, in accordance with the approval of the Local Ethical Committees, within the framework of a multicenter prospective cohort study, and funded by the Italian Ministry of Health (GR-20102317593). Traumatic meniscal tissue samples from the inner superficial zone were harvested from 5 patients with symptomatic meniscal tears undergoing arthroscopic partial meniscectomy (males, median age 41 years with interquartile range (IQR) of 4142 ; median of the body mass index (BMI) of $27.6 \mathrm{Kg} / \mathrm{m}^{2}$ with IQR of $\left.24.5-27.8\right)$. Endstage OA (Kellgren-Lawrence grades 3-4) tissue samples of meniscus were harvested from 3 patients undergoing total knee replacement (TKR) (2 males and 1 female, median age 72 years with IQR of 72-73.5). All the 5 patients selected for the study sustained a knee sprain during recreational sports activities ( 2 soccer; 2 running; 1 sailing). Specimens were collected after informed patient consent was obtained from each subject. As control, fragments from 3 multi-organ donors without a history of joint disorders (two males and one female, median age 66 years with IQR of 63-69) were collected from the Musculoskeletal Tissue Bank (IRCCS Istituto Ortopedico Rizzoli, Bologna, Italy). The meniscal injuries were evaluated by MRI before surgery. The meniscal injury was also confirmed by the surgeon during arthroscopy and described according to Trisolino et al. ${ }^{14}$ For patients undergoing arthroscopic partial meniscectomy pre- and post-operative symptoms were evaluated using the Knee Injury and Osteoarthritis Outcome Score (KOOS) total.

The meniscal samples used in the study were collected from multi-organ donors. Routinely, the fresh menisci are prepared by the Musculoskeletal Tissue Bank of our Institution for allograft and used for meniscal transplantation. The IRCCS Istituto Ortopedico Rizzoli (Bologna, Italy) is one of the leading institutions all around the world for this kind of surgery. Correct sizing is one of the most important factors in the success of the transplant. A screening process is done before selecting a possible donor and once selected the donor tissue undergoes many tests. The safety of the tissue is monitored and it is tested for viruses like those that cause HIV/AIDS, West Nile virus, hepatitis $\mathrm{B}$ and $\mathrm{C}$, as well as for bac- teria. The rate of discarded joint tissues is very low and only in this case those precious tissues are used for research. Moreover, the Italian law and the IOR ethical committee do not allow for tissue sampling from donors for research purposes; so we can obtain fresh samples from donors

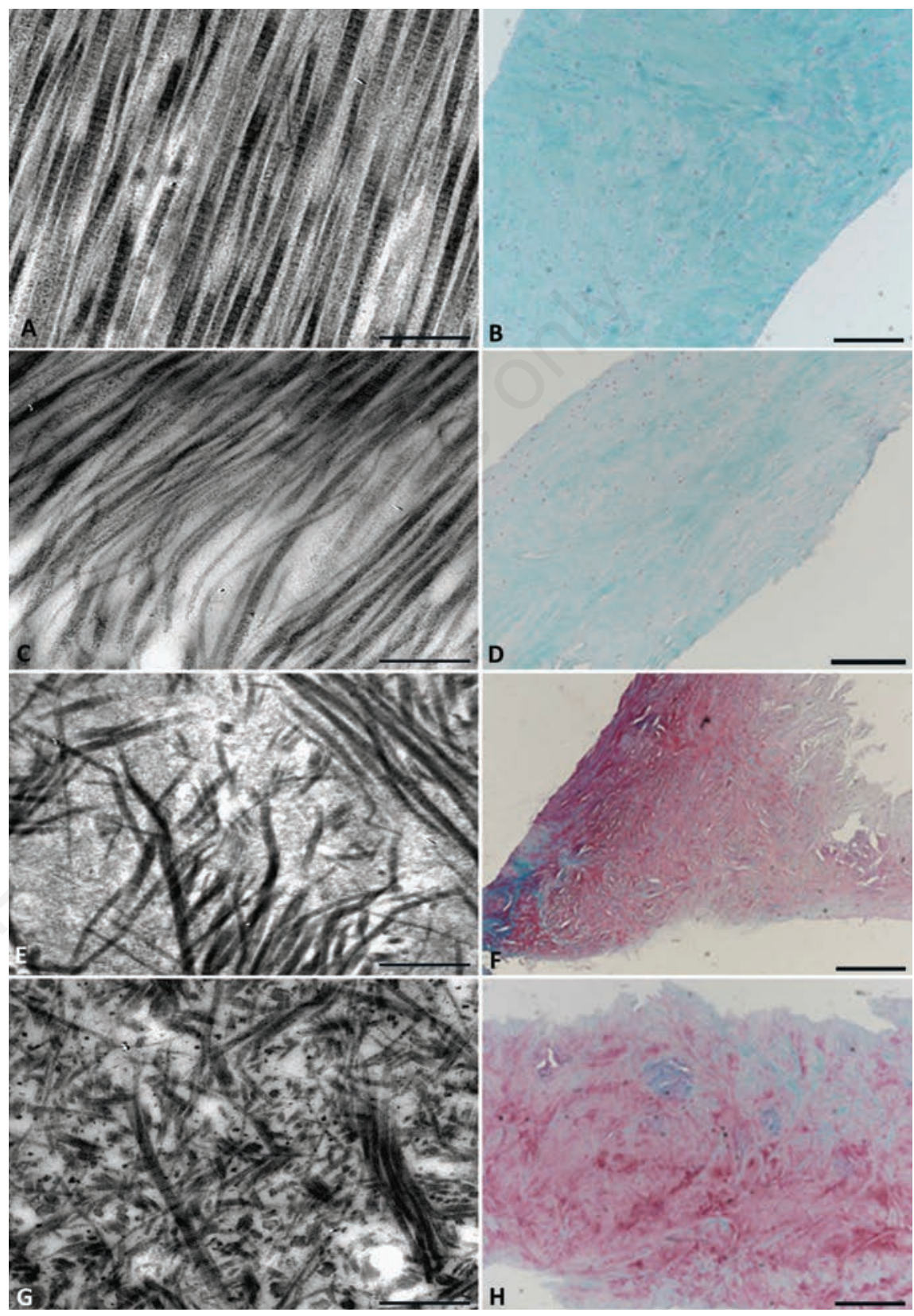

Figure 1. Meniscal tissue morphology. Meniscal samples collected from one representative multi-organ donor showed collagen fibers with homogenous distribution and orientation, as observed by TEM (A) and Safranin O/Fast green staining (B). Meniscal samples collected from a 25-year-old patient during the arthroscopic procedure for meniscal tear showed collagen fibers with regular size and distribution $(C)$ and virtually no staining for proteoglycans $(\mathrm{D})$. In samples from one representative patient with meniscal tear (53 years of age) (E and $F)$, and one representative patient with end-stage $O A$ ( $G$ and $H)$, TEM revealed collagen fibers with a structural disorganization of the ECM and strong staining for proteoglycans. Scale bars: A,C,E,G) $1 \mu \mathrm{m}$; B,F,H) $500 \mu \mathrm{m}$; D) $200 \mu \mathrm{m}$. 
only in case they were not used during the operation after assignment.

\section{Histological analyses}

Samples of meniscus from all subjects were fixed in 4\% formaldehyde (Kaltek, Padua, Italy) for histological examination. After fixation, tissues were embedded in paraffin, and sections of $5 \mu \mathrm{m}$ were prepared. The sections were stained with Gill III hematoxylin-eosin (Bioptica, Milan, Italy) and $0.25 \%$ Safranin O $/ 0.3 \%$ Fast Green (Sigma Aldrich, St Louis, MO, USA) to evaluate the general morphology and proteoglycan and observed at 10x magnification. The histological meniscal specimens' degeneration grade was assessed by a Pauli's microscopic grading system, which is validated to evaluate changes in three separate areas (femoral and tibial side and inner border) of aging and $\mathrm{OA}$ menisci. ${ }^{8}$ In our study, we modified Pauli's score because the meniscal biopsies were taken only from the inner border. The range of possible total scores was $0-12$, was converted into 4 grades: $\mathrm{G} 1=0-2, \mathrm{G} 2=3-5, \mathrm{G} 3=6$ 9 , and $\mathrm{G} 4=10-12$. Grade 1 represents normal tissue, Grade 2 is mild degeneration, Grade 3 is moderate degeneration, and Grade 4 is severe degeneration. To evaluate calcification in meniscal samples, the sections were stained with $1.4 \%$ Alizarin Red at $\mathrm{pH} 4.2$ (Sigma A5533). All images were captured with a Nikon Eclipse 90i microscope equipped with Nikon Imaging Software elements.

\section{Transmission electron microscopy (TEM)}

Samples were fixed with $2.5 \%$ glutaraldehyde in $0.1 \mathrm{M}$ cacodylate buffer for 3 $\mathrm{h}$, incubated with $1 \% \mathrm{OsO}_{4}$ in the same buffer for $1 \mathrm{~h}$, alcohol dehydrated, and embedded in araldite, as reported previously. ${ }^{15}$ Thin sections were collected on 400 mesh nickel grids and stained with uranyl acetate and lead citrate.

The observations were carried out with a Philips CM 10 electron microscope at 80 $\mathrm{kV} .{ }^{16}$ A qualitative analysis of the ECM fea- tures was performed on meniscal tissues, as described in Olivotto et al. ${ }^{17}$ To measure the collagen fiber diameter, we carried out a careful analysis of 20 sections for each sample, recording observations in 10 different fields for each section. ${ }^{18}$

A qualitative analysis was performed to identify viable cells and cells undergoing different types of cell death, known to occur in fibrochondrocytes. As described in Olivotto et al., ${ }^{17}$ cells were considered "viable" when both the nuclear and cytoplasmic membranes appeared intact and euchromatin was present. A cell was "nonviable" when the plasma membrane or nuclear membrane appeared fragmented

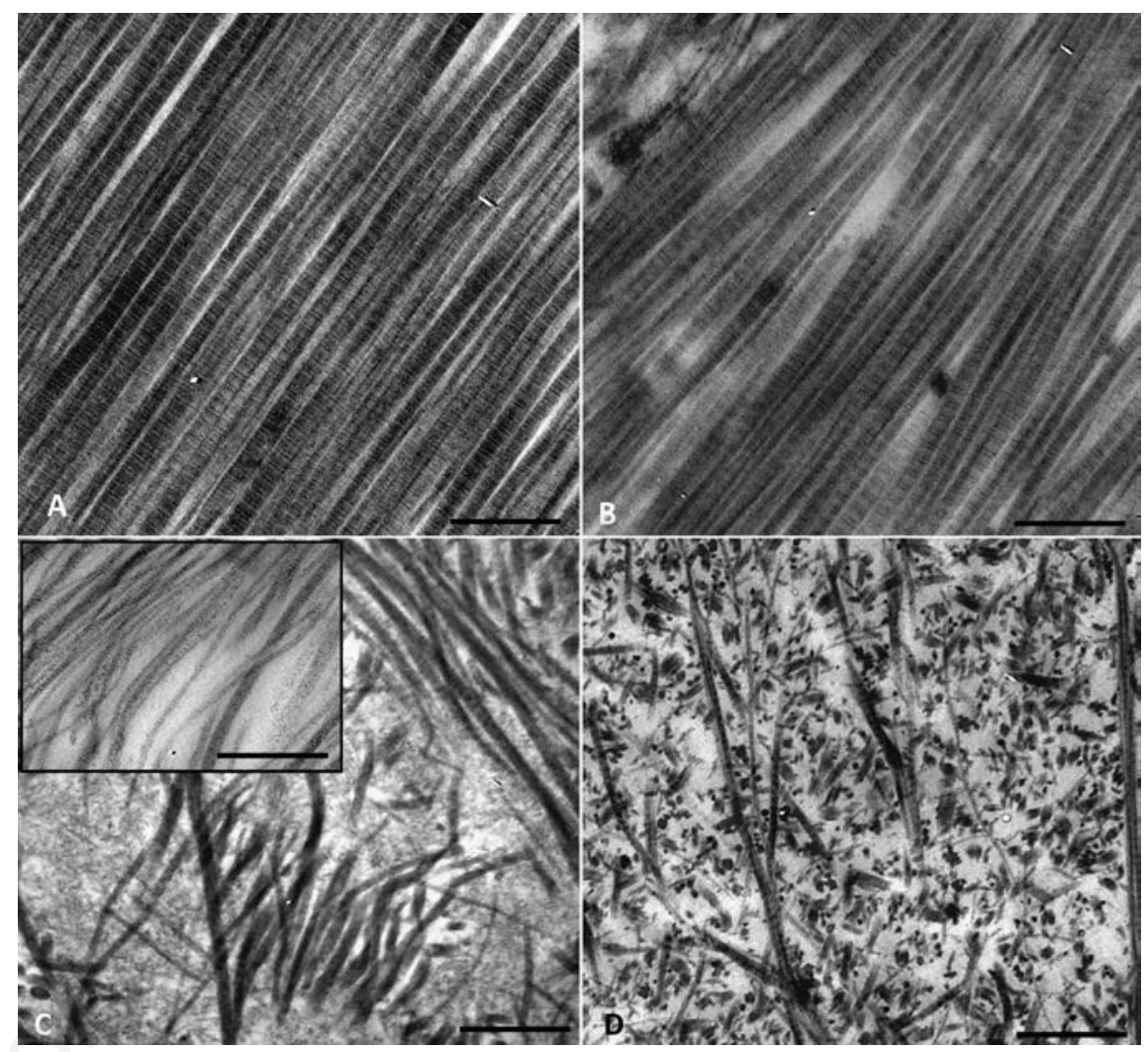

Figure 2. Collagen fiber organization and diameter. In samples from one representative multi-organ donor, the collagen fibers of the inner zone varied from 70 to $80 \mathrm{~nm}$ and showed the characteristic periodicity of collagen fibrillar organization (A). A meniscus biopsy collected from the 25-year-old patient undergoing arthroscopy for meniscal tear showed regular distribution of collagen fibers, appearing similar to that in the multiorgan donor (B). The sample from the 53-year-old patient showed meniscal degeneration with disorganized collagen fibers of between 35 to $45 \mathrm{~nm}$ in diameter ( $C$ and insert). The representative $\mathrm{OA}$ patient showed disorganized collagen fibers with diameters varying from 35 to $45 \mathrm{~nm}$ (D). Scale bars: $1 \mu \mathrm{m}$.

Table 1. Components of Pauli's meniscal degeneration score (surface, cellularity, collagen organization and Safranin O/Fast green staining) and total degeneration score.

\begin{tabular}{|c|c|c|c|c|c|c|}
\hline Pathology & Patient \# & Surface & Cellularity & Collagen & Saf-0/FG & Total score (grade) \\
\hline Meniscal tear & $\begin{array}{l}\# 1 \\
\# 2 \\
\# 3 \\
\# 4 \\
\# 5\end{array}$ & $\begin{array}{l}1 \\
1 \\
1 \\
1 \\
1\end{array}$ & $\begin{array}{l}0 \\
0 \\
0 \\
0 \\
0\end{array}$ & $\begin{array}{l}3 \\
2 \\
3 \\
2 \\
2\end{array}$ & $\begin{array}{l}3 \\
2 \\
3 \\
2 \\
2\end{array}$ & $\begin{array}{l}7 \text { (moderate) } \\
5 \text { (mild) } \\
7 \text { (moderate) } \\
5 \text { (mild) } \\
5 \text { (mild) }\end{array}$ \\
\hline Osteoarthritis & $\begin{array}{l}\# 1 \\
\# 2 \\
\# 3\end{array}$ & $\begin{array}{l}3 \\
3 \\
2\end{array}$ & $\begin{array}{l}0 \\
0 \\
0\end{array}$ & $\begin{array}{l}2 \\
3 \\
3\end{array}$ & $\begin{array}{l}2 \\
3 \\
3\end{array}$ & $\begin{array}{l}7 \text { (moderate) } \\
9 \text { (moderate) } \\
8 \text { (moderate) }\end{array}$ \\
\hline
\end{tabular}

Total score: G1, 0-2 normal; G2, 3-5 mild; G3, 6-9 moderate; G4, 10-12 severe; Saf-O/FG, Safranin O/Fast green staining. 
(which is indicative of necrosis or apoptosis, respectively).

\section{Results}

\section{Extracellular matrix changes}

Careful visual inspection of more than 10 TEM fields for the presence of organized ECM (including collagen fibers, fibrils, and proteoglycans) in all meniscal samples collected from multi-organ donors showed homogenous distribution and orientation of collagen fibers. The inner zone showed a high prevalence of collagen fibers with diffuse proteoglycans (Figure 1A), which was confirmed by light microscopy showing low staining intensity for Safranin O (Figure 1B). In general, after trauma, collagen fibers were reduced and proteoglycans increased (Figure 1C-F). Meniscal samples collected during the arthroscopic procedure for meniscal tear showed mild (3 patients) to moderate (2 patients) degeneration, assessed by the total of components of Pauli's meniscal degeneration score: surface, cellularity, collagen organization, and Safranin $\mathrm{O} /$ Fast green staining, as listed in Table 1. In the 25-year-old patient examined after trauma, the collagen fibers, which showed more regular size and distribution, were more abundant than proteoglycans (Figure $1 \mathrm{C}, \mathrm{D})$. In the other 4 patients, median age 45 years (Figure $1 \mathrm{E}, \mathrm{F}$ ), and in subjects with OA (Figure $1 \mathrm{G}, \mathrm{H}$ ), TEM observations, supported by histology (Figure $1 \mathrm{H}$ ), revealed the strong prevalence of proteoglycans compared to collagen fibers and structural disorganization of the ECM. Interestingly, among those patients with meniscal tear, the 53-year-old patient showed the worst meniscal degeneration with ECM loss. The collagen fiber diameter was also analyzed in all samples. In the inner zone of the multi-organ donor samples, the collagen fibers were aligned with a regular pattern for both distribution and diameter, which varied from 70 to $80 \mathrm{~nm}$ (Figure 2A), as described in the literature for the standard collagen fibers. ${ }^{19}$ Periodic collagen organization, mainly collagen Type II, which is the major fibrillar component of the inner-zone, was observed. Interestingly the meniscal biopsy collected from the 25-year-old patient, who had undergone arthroscopic surgery 2 years after the injury, showed the most regular distribution of collagen fibers, appearing similar to that in tissue from the multi-organ donor. Moreover, the fiber diameter appeared similar to those in the control samples from the multi-organ donors (Figure 2B). TEM observations showed that the other 4 patients with meniscal tears, in which the surgery was performed within 8 months from the injury, had structural disorganization of the ECM, including loss of collagen fiber organization. The collagen fibers were disorganized and the fiber diameter was inhomogeneous within the same specimen. The fiber diameter was between 45 and $60 \mathrm{~nm}$ (Figure 2C). Among those patients, the 53-year-old patient showed the worst meniscal degeneration with a collagen fiber diameter between 35 and $45 \mathrm{~nm}$ (Figure 2C, inset). As expected, OA patients showed disorganized collagen fibers and the fiber diameter varied from 35 to $45 \mathrm{~nm}$ (Figure 2D).
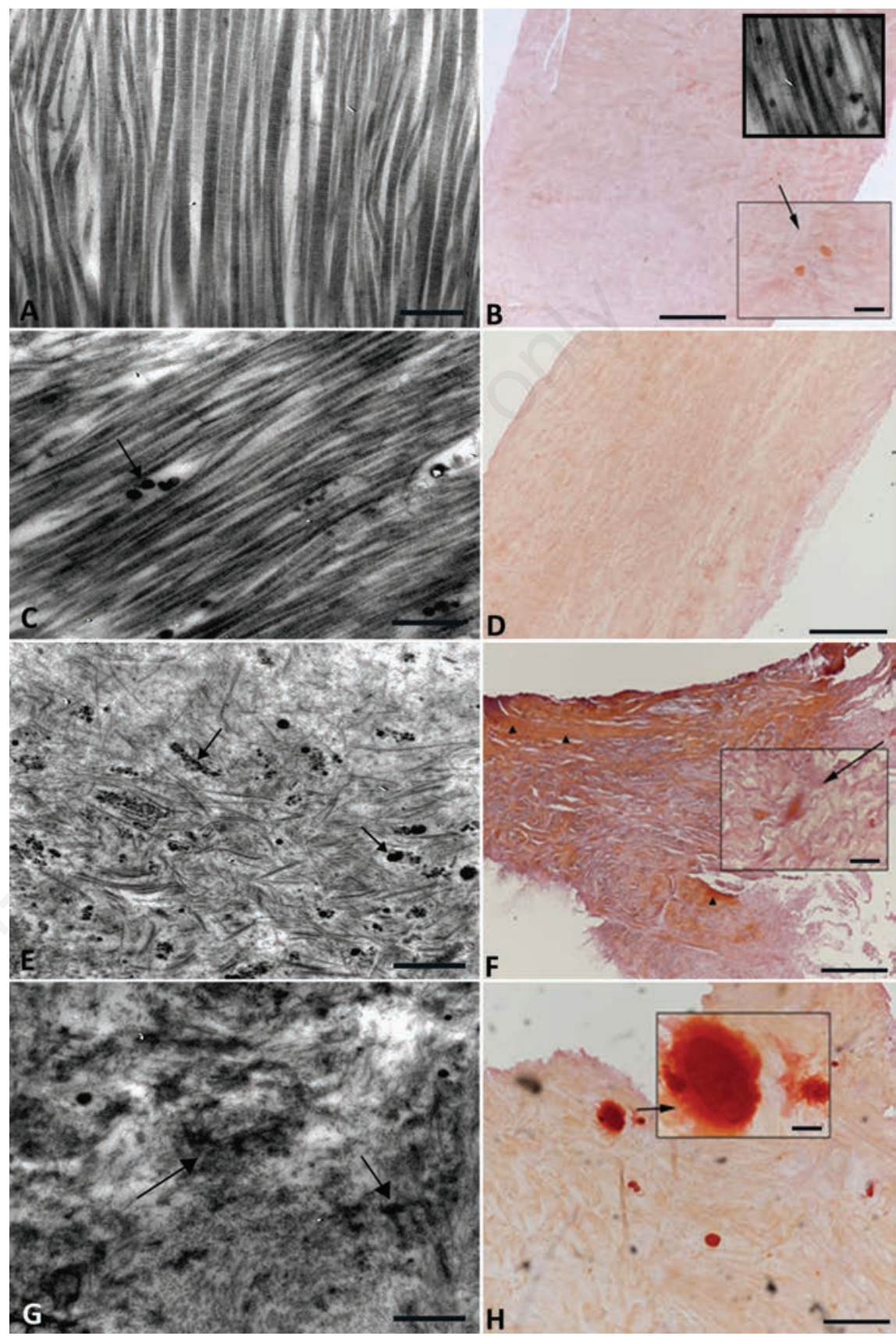

Figure 3. Calcium deposition. Meniscal samples collected from one representative multiorgan donor showed small calcium deposits, evaluated by Alizarin red staining (A) and rarely observed with TEM (B and insert). In meniscus samples collected from the 25-yearold patient, calcium deposits were not visible with Alizarin red (C) after trauma, but a few calcifications were detected by TEM (D). Large calcium deposits were observed in the 53-year-old patient ( $\mathrm{E}$ and $\mathrm{F}$ ). Extensive calcified areas were observed in one representative OA patient (G and H). Scale bars: A,C,E,G) $500 \mathrm{~nm}$; B,F,H) $500 \mu \mathrm{m}$; D) $200 \mu \mathrm{m}$; insert) $50 \mu \mathrm{m}$. 


\section{Calcium deposits}

Meniscal samples were evaluated for calcium deposits by TEM and Alizarin red staining. Among those collected from multiorgan donors, we observed by TEM rare calcium deposits (Figure 3A) and small calcium deposits (Figure 3B, inset) revealed by histology only in one patient. In general, after trauma we observed the presence of calcium deposits in all patients (Figure $3 \mathrm{C}$ F). Interestingly, in the 25-year-old patient with traumatic injury, few calcifications were detected by TEM and histology (Figure $3 \mathrm{C}, \mathrm{D})$, contrary to the other patients with meniscal tear (Figure $3 \mathrm{E}, \mathrm{F}$ ). In addition, we observed large calcium deposits only in the 53-year-old trauma patient. As expected, we observed extensive calcified areas in meniscal samples from all subjects with OA (Figure $3 \mathrm{G}, \mathrm{H}$ ).

\section{Cell morphology}

In the menisci of multi-organ donors, the cells embedded in the ECM showed rounded healthy morphology, characteristic of viable and metabolically active cells. The nuclei exhibited diffuse chromatin with small condensed areas near the nuclear membrane, both in the chondroblast-like cells (Figure 4A) and in those more similar to fibrochondrocytes (Figure 4B). The cytoplasm contained a high amount of glycogen, mitochondria were round and swollen, and the rough endoplasmic reticulum was well preserved. We observed small autophagic vacuoles (Figure 4 A,B).

In the young patient with traumatic meniscal tear, the chromatin showed small condensed areas (Figure 4 C,D), similar to the chromatin in the multi-organ donor, whereas small rare vacuoles and autophagic vacuoles could be observed in the cytoplasm (Figure $4 \mathrm{C}, \mathrm{D}$ ). In the older patients with traumatic meniscal tears, the nuclear chromatin appeared to be more condensed (Figure 4 E,F) compared to that in the healthy menisci of the multi-organ donors. Chromatin margination and condensation with a specific pattern, termed by Roach et $a l .{ }^{20}$ as "chondroptosis", was present in all samples from patients with traumatic injury (Figure 4). In the cytoplasm we observed abundant vacuoles but sparse cytoplasmic organelles, swollen and emptied mitochondria embedded in a relatively empty matrix (Figure 4 E,F). Autophagic vacuoles, presumably due to oxidative stress, were also present in the cytoplasm (Figure 4 E,F). In subjects with OA we observed large areas of condensed chromatin (Figure 4G) and large vacuoles within the cytoplasm (Figure $4 \mathrm{H}$ ).

\section{Clinical data}

In all patients with meniscal tear (examples of the macroscopic findings are shown
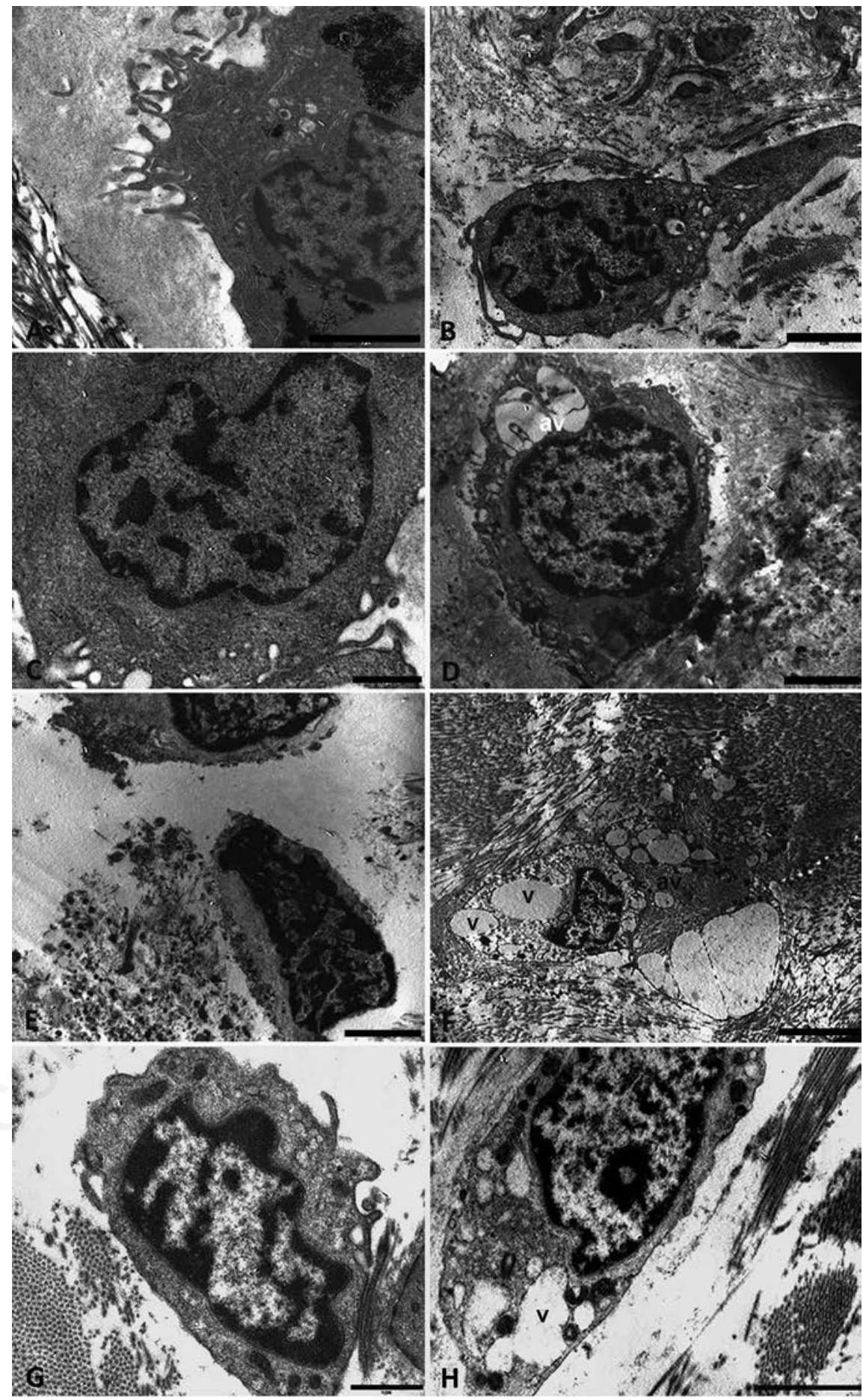

Figure 4. Cell morphology. In the menisci from multi-organ donors, the cells embedded in the ECM were round and showed a healthy morphology, consistent with viable and metabolically active cells. In the nucleus, the chromatin appeared diffuse with small condensed areas near the nuclear membrane (A and $B)$. In the young patient the chromatin showed small condensed areas $(C$ and $D)$, that were similar to those in the multi-organ donors with the cytoplasm containing small rare vacuoles (v) and autophagic vacuoles (av). In the older patients with traumatic meniscal tear meniscal cells contained nuclear chromatin that was more condensed than in the donor cells, with a specific pattern, called "chondroptosis". In the cytoplasm abundant vacuoles were present with scarcity of cytoplasmic organelles and swollen and emptied mitochondria (E, F). Autophagic vacuoles, consistent with oxidative stress, were also present in the cytoplasm. In the representative OA patients, large areas of condensed chromatin (G) and large vacuoles within the cytoplasm were observed (H). Scale bars: A) $2 \mu \mathrm{m}$; B-H) $1 \mu \mathrm{m}$. 
in Supplementary Figures 1 and 2) we observed a post-operative improvement of the KOOS at 1 and 2 years after arthroscopy (data not shown).

Interestingly, the 25-year-old patient also showed post-operative improvement despite having surgery 2 years after the injury.

\section{Discussion}

The analyses performed in our study indicate that different histological and morphological changes occur in the menisci from traumatic and end-stage OA patients that can be attributed to the different pathological conditions compared to the healthy donors. Meniscus samples collected from multi-organ donors showed low staining intensity for Safranin O, consistent with the median age of the donors. As described in Pauli et al., ${ }^{8}$ the major changes attributed to age included increased Safranin-O staining intensity. Despite early signs of aging, the morphological analysis revealed highly organized ECM with intact collagen fibers having periodic collagen organization and homogenous distribution and orientation. Contrary to Pauli's observation, ${ }^{8}$ we did not note any decrease in cell density in the donor samples and the morphological analysis showed healthy and active cells. Consistent with reports that calcifications occur in the menisci of $20 \%$ of elderly people without a history of joint disorders, ${ }^{21,22}$ we observed small calcium deposits in only one donor.

Meniscal biopsies collected from patients with meniscal tear showed similar features to those in OA patients, as described by Pauli et al. ${ }^{8}$ even though they were collected from injured patients of median age of 41 years, who were about 30 years younger than the patients with endstage OA. Of importance, the features of both groups were very different from those of the menisci from the healthy organ donor controls. The histological analysis of meniscal samples showed fibrocartilage disruption in patients with both meniscal tear and end-stage OA. These samples were characterized by proteoglycans increase and loss of structure with bands of degenerated ECM and with mostly disorganized collagen fibers. Evidence of increased proteoglycan deposition was shown by a moderate to strong staining intensity for Safranin-O. Interestingly, in contrast to meniscal samples from OA patients, the surface of those collected from patients with meniscal tear was often intact and there were distinct changes in ECM organization. This pattern is a typical age-related meniscal change in knees without history of major trauma. ${ }^{8}$
It is well established that meniscal tear is a risk factor for the development of OA. ${ }^{23}$ Among the mechanisms proposed, the release of inflammatory mediators in the space joint may trigger or accelerate the degenerative processes in the joint tissues, including the meniscus, ${ }^{2,24}$ as we observed in our study. Another factor is the duration of symptoms before the arthroscopic procedure is performed. Although a short duration of symptoms is one of the clinical variables that orthopedic surgeons consider, the evidence of the impact on clinical outcome is scarce. ${ }^{25}$ For example, Eijgenraam et al. found moderate evidence that duration of symptoms of longer than 3-12 months is associated with worse clinical outcome following meniscectomy. ${ }^{25}$

Our histological and morphological analysis showed that the meniscal degeneration is more related to the age of the individual rather than the duration of symptoms. In the 25-year-old patient, in spite of the 2 years of symptom duration before the arthroscopic procedure for partial meniscectomy, the features of the meniscal samples were similar to those from the multiorgan donors; furthermore, post-operative improvement was comparable to that observed in patients treated within 12 months following the injury. Therefore, age appeared to be the strongest incident factor determining the pre- and post-operative outcome.

In conclusion the limitation of our study is the low sample number of patients; thus, further research involving larger numbers of patients per group would enhance the significance of data. However, our observations at microscopic and ultrastructural levels confirm that meniscal injury activates ECM degeneration in meniscal tissue, and that meniscal tears in middle-aged patients present features similar to the OA degenerated meniscus. This potentially could account for the poorer clinical outcomes observed in treating older patients, thus suggesting the need for regenerative therapies to improve the results of treating traumatic lesions in middle-aged patients, as well as providing more information to support clinical treatment indications.

\section{References}

1. Makris EA, Hadidi P, Athanasiou KA. The knee meniscus: structure-function, pathophysiology, current repair techniques, and prospects for regeneration. Biomaterials 2011;32:7411-31.

2. Loeser RF, Goldring SR, Scanzello CR, Goldring MB. Osteoarthritis: a disease of the joint as an organ. Arthritis Rheum 2012;64:1697-707.
3. Hunter DJ, Zhang YQ, Niu JB, Tu X, Amin S, Clancy M, et al. The association of meniscal pathologic changes with cartilage loss in symptomatic knee osteoarthritis. Arthritis Rheum 2006; 54:795-801.

4. Ding C, Martel-Pelletier J, Pelletier JP, Abram F, Raynauld JP, Cicuttini F, et al. Meniscal tear as an osteoarthritis risk factor in a largely non-osteoarthritic cohort: a cross-sectional study. J Rheumatol 2007;34:776-84.

5. Englund M, Roos EM, Lohmander LS. Impact of type of meniscal tear on radiographic and symptomatic knee osteoarthritis: a sixteen-year followup of meniscectomy with matched controls. Arthritis Rheum 2003;48:217887.

6. Musumeci G, Loreto C, Carnazza ML, Cardile V, Leonardi R. Acute injury affects lubricin expression in knee menisci: an immunohistochemical study. Ann Anat. 2013;195:151-8.

7. Snoeker BA, Bakker EW, Kegel CA, Lucas C. Risk factors for meniscal tears: a systematic review including meta-analysis. J Orthop Sports Phys Ther 2013;43:352-67.

8. Pauli C, Grogan SP, Patil S, Otsuki S, Hasegawa A, Koziol J, et al. Macroscopic and histopathologic analysis of human knee menisci in aging and osteoarthritis. Osteoarthritis Cartilage 2011;19:1132-41.

9. Tsujii A, Nakamura N, Horibe S. Agerelated changes in the knee meniscus. Knee 2017;24:1262-70.

10. Thorlund JB, Englund M, Christensen R, Nissen N, Pihl K, Jorgensen U, et al. Patient reported outcomes in patients undergoing arthroscopic partial meniscectomy for traumatic or degenerative meniscal tears: comparative prospective cohort study. BMJ 2017;356:j356.

11. Hashimoto K, Oda Y, Nakamura F, Kakinoki R, Akagi M. Lectin-like, oxidized low-density lipoprotein receptor1-deficient mice show resistance to agerelated knee osteoarthritis. Eur J Histochem 2017;61:2762

12. Hashimoto K, Oda Y, Nakagawa K, Ikeda T, Ohtani K, Akagi M. LOX-1 deficient mice show resistance to zymosan-induced arthritis. Eur J Histochem 2018;62:2847

13. Yamagishi K, Tsukamoto I, Nakamura F, Hashimoto K, Ohtani K, Akagi M. Activation of the renin-angiotensin system in mice aggravates mechanical loading-induced knee osteoarthritis. Eur J Histochem 2018;62:2930.

14. Trisolino G, Favero M, Lazzaro A, Martucci E, Strazzari A, Belluzzi E, et al. Is arthroscopic videotape a reliable 
tool for describing early joint tissue pathology of the knee? Knee 2017;24:1374-82.

15. Battistelli M, Borzi RM, Olivotto E, Vitellozzi R, Burattini S, Facchini A, et al. Cell and matrix morpho-functional analysis in chondrocyte micromasses. Microsc Res Tech 2005;67:286-95.

16. Battistelli M, Salucci S, Olivotto E, Facchini A, Minguzzi M, Guidotti S, et al. Cell death in human articular chondrocyte: a morpho-functional study in micromass model. Apoptosis 2014;19: 1471-83.

17. Olivotto E, Borzi RM, Vitellozzi R, Pagani S, Facchini A, Battistelli M, et al. Differential requirements for IKKalpha and IKKbeta in the differentiation of primary human osteoarthritic chondrocytes. Arthritis Rheum 2008; 58:227-39.

18. Battistelli M, De Sanctis R, De Bellis R, Cucchiarini L, Dacha M, Gobbi P. Rhodiola rosea as antioxidant in red blood cells: ultrastructural and hemolytic behaviour. Eur J Histochem 2005;49: 243-54.

19. Raspanti M, Viola M, Sonaggere M, Tira ME, Tenni R. Collagen fibril structure is affected by collagen concentration and decorin. Biomacromolecules 2007;8:2087-91.

20. Roach HI, Aigner T, Kouri JB. Chondroptosis: a variant of apoptotic cell death in chondrocytes? Apoptosis 2004;9:265-77.

21. Mitrovic DR. Pathology of articular deposition of calcium salts and their relationship to osteoarthrosis. Ann Rheum Dis 1983;42:19-26.

22. Sun Y, Mauerhan DR. Meniscal calcification, pathogenesis and implications. Curr Opin Rheumatol 2012;24:152-7.

23. Lieberthal J, Sambamurthy N, Scanzello CR. Inflammation in joint injury and post-traumatic osteoarthritis. Osteoarthritis Cartilage 2015;23:182534.

24. Scanzello CR, Goldring SR. The role of synovitis in osteoarthritis pathogenesis. Bone 2012;51:249-57.

25. Eijgenraam SM, Reijman M, BiermaZeinstra SMA, van Yperen DT, Meuffels DE. Can we predict the clinical outcome of arthroscopic partial meniscectomy? A systematic review. $\mathrm{Br}$ J Sports Med 2018;52:514-21. 